\title{
Research on the Optimal Conditions of the Polysaccharide Extraction from Pholiota Nameko
}

\author{
Zang Yu-Hong \\ The Chemical Engineering Department, Chengde Petroleum College, Chengde, Hebei, China \\ 594241891@qq.com.cn
}

\begin{abstract}
The purpose of the present study was to investigate the optimal process of the polysaccharide extraction from Pholiota nameko. The pholiota nameko fruiting bodies was used as raw material to extract crude polysaccharide by water and ethyl alcohol. The extraction process of Pholiota nameko polysaccharide was optimized by single factor and orthogonal experiments. The experimental results show that the optimum technological conditions were solid-liquid ratio 1:40, temperature $70^{\circ} \mathrm{C}$, the extraction time 5.5 hours, the concentration of ethanol $80 \%$. under above conditions , the crude polysaccharide extraction yield could be up to $6.23 \%$.
\end{abstract}

Index Terms - pholiota nameko, extraction technology, polysaccharide

\section{Foreword}

Pholiota nameko is also known as the slider mushroom, light cap Pholiota adiposa, pearl beauty etc. Because the slider mushroom fruiting body surface covered with mucus, touch stick slip, so it is named of Pholiota nameko. It is a kind of wood saprophytic, full of nutrition, and contains a variety of minerals [1].

Edible fungi polysaccharides have antiviral, antitumor [2], regulate immune function and anti-infection activities [3], as well as enhance the immune system [4], act as antioxidant and lower blood sugar. They also have biological activities and physiological functions of anti-ulcer, anti-aging and anti-radiation etc. They can prevent rickets and cold, and also have good effect to reduce serum cholesterol [5]. At present, many researches are about Pleurotus ostreatus polysaccharide [6], Flammulina velutipes polysaccharide [7] and Auricularia auricula polysaccharide [8], etc; the nameko polysaccharide was rarely reported. In this paper, the optimum conditions of Pholiota nameko polysaccharide extraction were explored, in order to open up new ways for deep processing of Pholiota nameko.

\section{Materials and methods}

\subsection{Raw materials and reagents}

Pholiota nameko and chemical regents like hydrochloric acid, sodium hydroxide, n-buty alcohol, ethanol (95\%), chloroform, anthrone, sulphuric acid and glucose etc. were used, and they are all of analytical grade.

\subsection{The main instrument}

In the experiment, instrument like 101-2 type oven, organization stamp mill, 80-2B centrifuge, HH-6-type constant temperature water bath, $\mathrm{pHS}-3$ precise $\mathrm{pH}$ meter, SHB-B vacuum filtration machine, KDM electric sets, 721 and 722 spectrophotometer, JY3002 electronic balance and etc. have been employed.

\subsection{Experimental Methods}

2.3.1 Preparation of standard curve: The polysaccharide standards dried to constant mass at $120{ }^{\circ} \mathrm{C}$ oven, and Cooled in the desiccator, then The 100mg standards was dissolved in deionized water and set the volume 500mL.A series of different concentrations of glucose solution was added anthrone reagent, boiling water bath heating $10 \mathrm{~min}$, cooling. Light absorption value was measured at $490 \mathrm{~nm}$,We drew standard curve with the standard concentration as the abscissa, the absorption value as the ordinate.

2.3.2 Pretreatment: First select Pholiota nameko, and then they were washed and dried for future use.

2.3.3 The second step is crushing, taking a certain amount of Pholiota nameko, crushed and sieved.

2.3.4 The single factor experiment: We select extraction temperature, extraction time and solid-liquid ratio as parameters for the single factor experiment.

2.3.5 The optimized process: On the basis of the single factor experiment, the orthogonal experiment $\mathrm{L}_{9}\left(3^{4}\right)$ was designed to determine the optimal conditions.

2.3.6 The verification experiment: To confirm that the experiment get the highest yield under the optimum conditions.

3.3.7 The polysaccharide yield calculation:

$$
\begin{aligned}
& Y / \%=\frac{D V X}{W \times 10^{6}} \times 100 \% \\
& X=\frac{O D{ }_{490}-0.0115}{0.074}
\end{aligned}
$$

$\mathrm{X}$ - nameko polysaccharide concentration $(\mathrm{g} / \mathrm{ml})$; D- nameko polysaccharide sample dilution factor; $\mathrm{V}$ - nameko polysaccharide sample liquid volume $(\mathrm{ml})$; W-Pholiota nameko sample dry weight (g)

\section{Results and Discussions}

\subsection{Standard curve}

Standard curve was drawn by using anthrone - sulfuric acid method. The result is shown in Figure 1. 


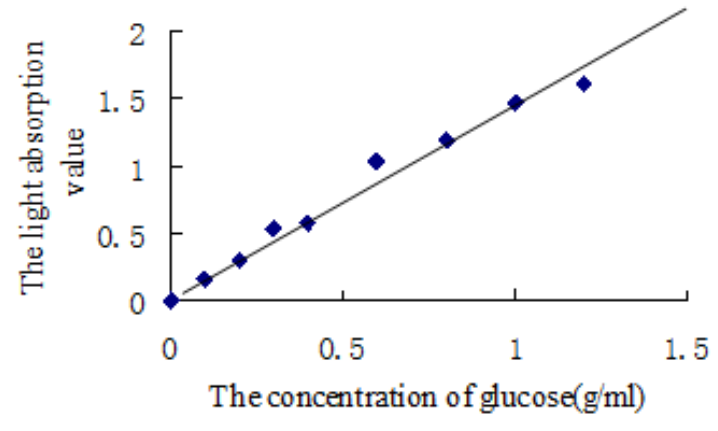

Fig. 1 Standard curve of sugar

\subsection{Results of single factor experiment}

\subsubsection{Effect of solid-liquid ratio on extraction yield}

When we kept other parameters constant, we tested the relation between Pholiota nameko polysaccharide extraction yield and solid-liquid ratio and the results are shown in Figure2.

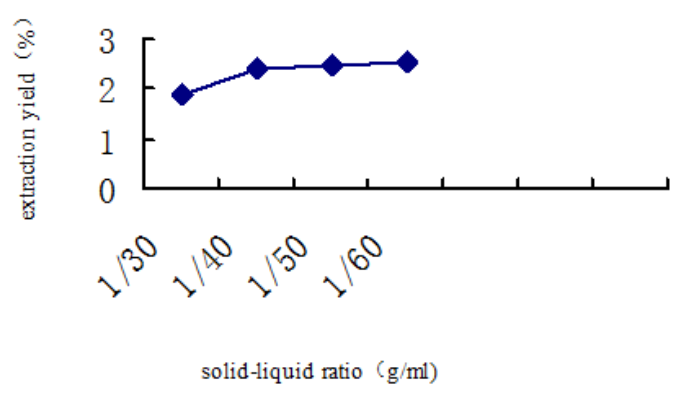

Fig. 2 Effect of solid-liquid ratio on extraction yield

From the analysis of the data in Figure 2, we know that nameko polysaccharide extraction rate improves with the increase of solid-liquid ratio. However the increase of extraction yield became relatively slow when the solid-liquid ratio is more than 1:40. In order to facilitate follow-up water treatments, we set the solid-liquid ratio to 1:40.

\subsubsection{Effect of extracting time on extraction yield}

By the analysis of data in Figure 3, extraction rate reached to the maximum value when extraction time was 5 $\mathrm{h}$. Therefore extraction time $5 \mathrm{~h}$ is the best time of polysaccharide extraction.

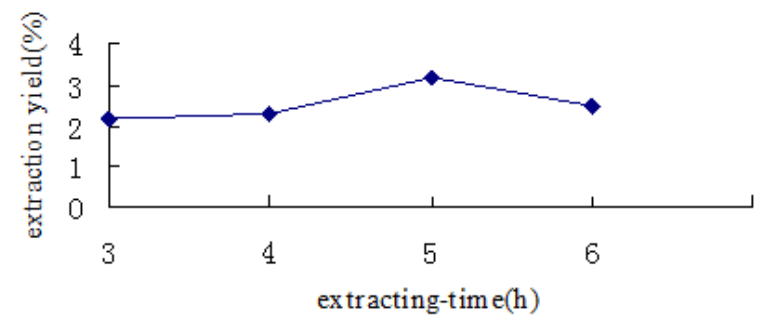

Fig. 3 Effect of extracting-time on extraction yield

\subsubsection{Effect of temperature on extraction yield}

By the analysis of data in Figure 4, extraction rate reached the maximum value when extraction temperature is $80{ }^{\circ} \mathrm{C}$. However at temperature range of $70{ }^{\circ} \mathrm{C} \sim 90{ }^{\circ} \mathrm{C}$, the extraction yield underwent slight changes and further temperature increase even caused a decreased extraction yield. From the viewpoint of energy saving, polysaccharide extraction temperature was selected $70{ }^{\circ} \mathrm{C}$.

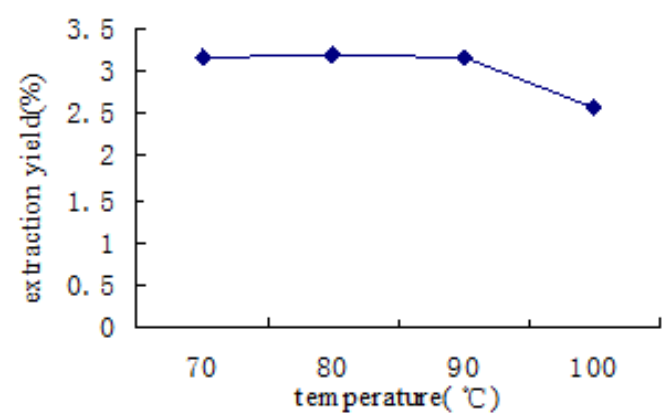

Fig. 4 Effect of temperature on extraction yield

\subsection{The results of orthogonal experiments}

The design of orthogonal experiment was based on single factor experiment to optimize extraction process of Pholiota nameko polysaccharide. The design is shown as Table1.

Table 1 The design of orthogonal experiment $\mathrm{L}_{9}\left(3^{4}\right)$

\begin{tabular}{|c|c|c|c|c|}
\hline Level & solid-liquid ratio & temperature & time & concentration of ethanol \\
\hline & $\mathrm{A}(\mathrm{g} / \mathrm{ml})$ & $\mathrm{B}\left({ }^{\circ} \mathrm{C}\right)$ & $\mathrm{C}(\mathrm{h})$ & $\mathrm{D}(\%)$ \\
\hline 1 & $1: 20$ & 60 & 5 & 70 \\
\hline 2 & $1: 30$ & 70 & 5 & 80 \\
\hline 3 & $1: 40$ & 80 & 5.5 & 90 \\
\hline
\end{tabular}

Results and analysis of Orthogonal Experimental are shown in Table 2

From the analysis of the data in Table 2, we know that the optimum combination of the technological conditions was $\mathrm{A}_{3} \mathrm{~B}_{2} \mathrm{C}_{3} \mathrm{D}_{2}$, i.e. solid-liquid ratio was $1: 40$; the temperature was $70^{\circ} \mathrm{C}$, the extraction time was 5.5 hours and the concentration of ethanol was $80 \%$. By the analysis of the importance of factors, we know that for extraction conditions, the importance of the influencing factor follow the order of $\mathrm{B}>\mathrm{D}>\mathrm{A}>\mathrm{C}$, namely, temperature > ethanol concentration $>$ solid-liquid ratio $>$ extracting time. 
Table 2 The result and analysis of orthogonal experiment $\mathrm{L}_{9}\left(3^{4}\right)$

\begin{tabular}{|cccccc|}
\hline no & $\mathrm{A}(\mathrm{g} / \mathrm{ml})$ & $\mathrm{B}\left({ }^{\circ} \mathrm{C}\right)$ & $\mathrm{C}(\mathrm{h})$ & $\mathrm{D}(\%)$ & yield $(\%)$ \\
\hline 1 & 1 & 1 & 1 & 1 & 3.976 \\
3 & 1 & 2 & 2 & 2 & 5.432 \\
4 & 1 & 3 & 3 & 3 & 4.480 \\
5 & 2 & 1 & 2 & 3 & 2.548 \\
6 & 2 & 2 & 3 & 1 & 4.640 \\
7 & 3 & 1 & 3 & 2 & 3.960 \\
8 & 3 & 2 & 1 & 3 & 5.400 \\
9 & 3 & 3 & 2 & 1 & 3.940 \\
$\mathrm{~K} 1$ & 13.888 & 11.924 & 11.876 & 13.26 & \\
$\mathrm{~K} 2$ & 11.15 & 14.012 & 12.62 & 14.79 & $\mathrm{R}=37.02$ \\
$\mathrm{~K} 3$ & 13.98 & 13.08 & 14.52 & 10.968 & \\
$\mathrm{k} 1$ & 4.627 & 3.975 & 3.959 & 4.420 & $\mathrm{~V}=4.113$ \\
$\mathrm{k} 2$ & 3.716 & 4.671 & 4.207 & 4.930 & \\
$\mathrm{k} 3$ & 4.660 & 4.360 & 4.840 & 3.656 & \\
$\mathrm{R}$ & 0.944 & 1.668 & 0.887 & 1.274 & \\
\hline
\end{tabular}

\subsection{Verification experiment}

The design of verification experiment was based the optimum extraction conditions, and the result is shown in Table 3.

Table 3 The result of the best extracting conditions

\begin{tabular}{|c|c|c|c|c|c|}
\hline No & solid-liquid ratio & temperature & time & yield & average yield \\
\hline 1 & $1: 40$ & $70^{\circ} \mathrm{C}$ & $5.5 \mathrm{~h}$ & 6.76 & \multirow{2}{*}{6.23} \\
\hline 2 & $1: 40$ & $70^{\circ} \mathrm{C}$ & $5.5 \mathrm{~h}$ & 5.65 & \multirow{2}{*}{6.23} \\
\hline 3 & $1: 40$ & $70^{\circ} \mathrm{C}$ & $5.5 \mathrm{~h}$ & 6.28 & \multirow{2}{*}{} \\
\hline
\end{tabular}

The result show that polysaccharide extraction yield was $6.23 \%$ in the optimum conditions.

\section{Conclusions}

The optimal conditions of the polysaccharide extraction from Pholiota nameko were that solid-liquid ratio was $1: 40$, the best temperature was $70^{\circ} \mathrm{C}$, the extraction time was 5.5 hours and the concentration of ethanol was $80 \%$. Extraction parameters influencing the yield followed the order of temperature > concentration of ethanol > solid-liquid ratio $>$ extracting time and the highest yield achieved was $6.23 \%$.

\section{References}

[1] ZANG Yu- hong, NIU Gui- ling, LI Li- juan, etc. Study on the extraction technology of water soluble polysaccharides from pholiota nameko. food science and technology ,2006,(11):115-118

[2] TONG Hai-bin, XIA Feng-guo, FENG Kai, et al. Structural Characterization and Invitro Antitumor Activity of Anovel Polysaccharide Isolated from the Fruiting Bodies of Pleurotus ostreatus. Bioresour Technol, 2009, 100(4):1682-1686

[3] WANG Xue-bing, ZHAO Tian-rui, FAN Jian. Mushroom Polysaccharide Extraction Technology Overview. edible fungi of china , 2010, 29(2):3-6

[4] LAI Fu-rao, WEN Qi-biao, LI Lin, et al. Antioxidant Activities of Water-soluble polysaccharide Extracted form Mungbean (vigna radiate L) Hull with Ultrasonic Assisted Treatment. Carbohydrate polymers, 2010, 81(2):323-329

[5] LIU Xiao-nan, ZHOU Bo, LIN Rong-shan, et al.Extraction and Antioxidant Activities of Intracellular polysaccharide form Pleurotus spMycelium. Int Biol Macromol, 2010, 47(2):116-119

[6] ZHANG Min, CHEN Rui-zhan, CHEN Rui-ping. Comparison of extraction methods of Polysaccharide from Pleurotus ostreatus. Journal of Jilin University Science Edition. 2011,49(6):1141-1148

[7] JIANG Hai-ming, ZHANG Xiu-hua. Flammulina velutipes polysaccharide extraction process research. Journal of Anhui Agricultural Sciences, 2011, 39(11):6524-6525.

[8] LU Wen, CAI Zhen-you, XIAN Qiao. Study on extraction conditions of Auricularia auricula polysaccharides. China Food Additives, 2010, (6):99-102. 